\title{
Diffuse Endocapillary Glomerulonephritis
}

National Cancer Institute

\section{Source}

National Cancer Institute. Diffuse Endocapillary Glomerulonephritis. NCI Thesaurus. Code C122806.

Glomerulonephritis characterized by endocapillary proliferation and the presence of inflammatory cells affecting 50\% or more of all glomeruli. 